\title{
Adsorbed Block Copolymer Layers: Assembly and Tailoring of Polymer Brushes
}

\author{
M. Tirrell, ${ }^{* * 1}$ E. Parsonage, ${ }^{* 1}$ H. Watanabe, ${ }^{* 2}$ \\ and S. DHOOT*1 \\ ${ }^{* 1}$ Department of Chemical Engineering and Mateirals Science, \\ University of Minnesota, 421 Washington Avenue S. E., \\ Minneapolis, Minnesota 55455 U.S.A. \\ *2 Department of Macromolecular Science, Osaka University, \\ Toyonaka, Osaka 560, Japan
}

(Received November 26, 1990)

\begin{abstract}
Measurements have been made of the forces of interaction between pairs of adsorbed layers of diblock copolymers. These have been made on layers of 2-vinylpyridine-styrene and 2-vinylpyridine-isoprene copolymers. From toluene solution, the vinylpyridine block adsorbs strongly and the other blocks adsorb negligibly. This paper discusses the adsorption process itself and ways in which the interactions between two such layers can be tailored for particular purposes.
\end{abstract}

KEY WORDS Adsorption /Block Copolymers/Surface Force Measurement/ Poly-2-vinylpyridine-polystyrene / Poly-2-vinylpyridine-polyisoprene /

Block copolymers in solution afford the opportunity to create unique adsorbed layers via selective adsorption from a selective solvent. This process uses the amphiphilic character of block copolymers in that it can be arranged that one block adsorbs strongly on a solid surface and avoids the solvent, while the other block, nonadsorptive on its own, swells into the good solvent surrounding medium but remains tethered to the surface by the adsorbing block. ${ }^{1-3}$ This provides a means to modify the interactions of solid surfaces immersed in solvents. One block of the copolymer anchors firmly while the other extends out and produces the desired interaction with the environment.

This self-assembly process has proven very useful in technology to modify the surfaces of colloidal particles that are used in coatings, paints, xerography, photography, and processes for ultrafine ceramics. ${ }^{4,5}$ The scientific questions that are posed in this situation concern the nature of the configurations adopted by the macromolecules in this environment and how peculiarities in this configuration may reflect themselves in the nature of the interactions that these layers produce with the surroundings.

The following facts appear to be established concerning the molecular arrangements in these layers. In the interesting case that the anchoring energy, $\varepsilon_{A} N_{A}$, is large, where $\varepsilon_{A}$ is the binding energy per anchoring segment and $\mathrm{N}_{\mathrm{A}}$ is the number of anchoring segments, the number of chains adsorbed per unit area, $\sigma \mathrm{a}^{-2}$, (with a being the size of a monomer unit) can be sufficiently high that it exceeds the threshold for overlap of the nonadsorbing chains (the "buoys"). This criterion for overlap of the buoys can be expressed as: 6

$$
\sigma_{\mathrm{ol}}=\mathrm{N}_{\mathrm{B}}-6 / 5
$$

where $N_{B}$ is the number of segments in the buoy block. Only if $N_{A} \gg N_{B}$, or if the anchoring energy is very weak, is this criterion not met. ${ }^{3}$ Under these circumstances, the buoy chains form a polymer "brush" 6-9, in which the chains extend well beyond their solution dimensions in the direction normal to the adsorbing plane. That a polymer brush 
should be extended can be understood from a Flory-type argument, balancing osmotic swelling of the dense brush with elastic resistance to swelling. In terms of the free energy per chain in the brush, F/kT, this argument can be written:

$$
\mathrm{F} / \mathrm{kT}=\mathrm{L}^{2} / 2 \mathrm{Na}^{2}+\mathrm{vN}(\mathrm{Na} \sigma / \mathrm{L})
$$

where $\mathrm{v}$ is a dimensionless excluded volume $(\sim 1-2 \chi)$ and the quantity in parentheses is the volume fraction of polymer segments in a uniformly stretched brush of height $\mathrm{L}$. Minimization of this energy with respect to L gives:

$$
\mathrm{L}=\mathrm{Na}(\mathrm{v} \sigma)^{1 / 3}
$$

The linear dependence of $\mathrm{L}$ on $\mathrm{N}$ signals that chains in a brush have characteristic dimensions that scale more like chains standing on end than random coils, though, as we shall demonstrate, typical values of $\sigma$ that are achieved by the examples of selective adsorption that have been tried so far are of order 0.01 , so that, $\mathrm{L}<<\mathrm{Na}$.

This equation has been tested and appears to agree reasonably well with the length scale of brushes as measured both by surface forces measurement and by neutron scattering. ${ }^{10,11}$ However, this should lead neither to the conclusion that the profile of segments is consistent with uniform stretching nor that Equation 2 is an accurate one for the stretching energy. It has been clearly demonstrated by self-consistent field calculations $^{12 \mathrm{a}}$ and by molecular dynamics simulations ${ }^{12 \mathrm{~b}}$ that the stretching of the chains is not uniform (decreasing with distance from the surface) and that chain ends, implicitly assumed in Equation 2 to be all at the tip of the brush, in fact, distribute themselves throughout the depth of the brush. The resulting profile of segments is more like parabolic 12 than uniform, though Equation 3 remains a good quantitative measure of the average brush height.

The reasons why Equation 2 does not give a good measure of the stretching energy, even though Equation 3 is adequate, have been discussed by de Gennes ${ }^{13}$ and by Freed ${ }^{14}$. The principal reason is that both terms in Equation 2 are overestimates that cancel in going to Equation 3. To get a more accurate calculation of the stretching energy, both terms must be modified to account for correlations present in the strong excluded volume environment in good solvent. For example, the osmotic pressure energy should be proportional to $(\mathrm{N} \sigma \mathrm{a} / \mathrm{L})^{5 / 4} 15$, instead of linear, since correlations reduce the number of binary contacts between segments. Using this to estimate the energy by substituting Equation 3 into the osmotic term of Equation 2 modified in this way gives a stretching energy per layer $(=$ energy per chain times $\sigma)$ :

$$
\mathrm{F}_{\text {layer }} / \mathrm{kT}=\mathrm{Nv}^{7 / 12} \sigma^{11 / 6}
$$

This equation represents the penalty that a chain must pay to adsorb in a high density brush. This will be the basis for the analysis of the adsorption data that we present in this paper.

The other aspect of polymer brushes that this paper will discuss is the means by which the profile of segments extending away from the surface can be tailored. In a sense, the nonuniform stretching recognized by the $\mathrm{MWC}^{12}$ theory, though clearly correct, is a disappointment to the extent that layers with all of the chain ends at the periphery may have special properties or utility. For example, if a synthetic reaction were designed to place a particular functional group at the ends of the chains this could be used to create a brush with an array of "stickers" at the tips of the bristles. The analysis of the polydisperse brush ${ }^{16}$ shows that ends of longer chains are segregated from the shorter, denser brush for essentially the same reason that brushes are impenetrable to other brushes or solutions of equal or lower segment density. It is more favorable for the extra chain length to reside above the dense brush than to penetrate it and cause the dense brush to stretch still more.

One means to tailor the profile of segments in a brush is to use a specified 
distribution of chain lengths in the assembly of the brush. A few longer bristles on the brush bearing the stickers may recover some of the desirable feature of the hypothetical uniformly stretched brush. Two questions (at least) arise in this connection. One is whether or not the competitive adsorption process strongly favors one block copolymer over another. We have and present evidence here for what the thermodynamically favored tendencies are. Kinetic favorization is also possible, even likely, though it is unstudied as yet in competitive adsorption. We report here on our first measurements on the self-assembly and forces exerted between bimodal brushes. These have been made on layers of 2-vinylpyridine-styrene (PVP-PS) and 2-vinylpyridine-isoprene (PVP-PI) copolymers. From toluene solution, the vinylpyridine block adsorbs strongly and the other blocks adsorb negligibly. ${ }^{1}$

\section{EXPERIMENTAL}

The methods we have used to determine the adsorbed amounts of PVP-PS and PVP-PI block copolymers adsorbed on mica from toluene have been described in detail in another publication. ${ }^{3}$ We discuss here only the most salient features, as well as the aspects of the method necessary to study bimodal brushes. All of the polymers were prepared by anionic polymerization and had molecular weights distributions with a polydispersity of less than 1.1. Table I lists all of the block copolymers on which we have measured adsorbed amounts. Surface forces measurements have been made on a subset of these. Several of these samples, those including a prefix t- in their sample designations, have been synthesized incorporating a radioactive label. This was done by terminating the living polymerization with tritiated methyliodide $\left(\mathrm{CT}_{3} \mathrm{I}\right)$. Scintillation counting on the carefully and completely collected combustion products from incineration of the adsorbed layer is then an accurate means of determining the weight of polymer adsorbed per unit area.

However, the method we have developed is more versatile than scintillation counting alone. X-ray photoelectron spectroscopy (XPS) is a very accurate method for the determination of the relative adsorbed amounts in two adsorbed layers. It does so by measuring the attenuation of the signal from a particular photoelectron $\left(\mathrm{Si}_{2 \mathrm{p}}\right.$ in the case of mica or silica) in the substrate. We have shown that for a series of chemically similar materials such as the range of composition represented in Table I, the escape depth for photoelectrons varies by only about $1 \AA$, so that the normalized signal from the attenuated photoelectron is directly proportional to the surface coverage. If the coverage of one or more of the polymers is known, the relative measure provided by XPS can be calibrated into a absolute method. Calibration with the independently determined adsorbed amounts of the tritiated polymers enables this to be done. In our hands the precision of determination of the adsorbed amounts by this method is approximately $\pm 5 \%$.

Scintillation counting also permits the determination of the composition in a mixed adsorbed layer containing two species of chains. In this case, the total adsorbed amount is determined by XPS and the fraction of a tritiated species in the adsorbed layer is determined by scintillation counting. If the internal copolymer compositions of the two species used to assemble the bimodal layer is sufficiently different, we have found that we can also determine the composition of the adsorbed layer directly from XPS by measuring the carbon to nitrogen elemental composition ratio of the adsorbed layer and using the known elemental compositions of the copolymers.

The methods used in our laboratory for surface forces measurement have also been described in detail previously ${ }^{10}$. We refer the interested reader there for details. The adsorption is done in all cases inside the apparatus under conditions otherwise identical to those under which the layers on which surface coverage was measured were assembled. All force curves reported are believed to represent equilibrium forces exerted at the indicated distances, with approaching force curves equivalent to separating force curves within experimental accuracy. As is convenient and conventional, all force versus distance data are plotted as F/R vs. $D$, where $R$ is the measured local radius of curvature of the mica sheets in the force-measuring zone, thereby converting the data into a quantity 


\section{Table I}

Summary of adsorbed amounts for PVP-PS and PVP-PI diblock copolymers. The final column is the surface density $\left(\sigma_{\mathrm{ol}}\right)$ above which the PS buoys will overlap in the adsorbed layers.

\begin{tabular}{|c|c|c|c|}
\hline $\begin{array}{l}\text { Sample } \\
\text { PVP-PS }\end{array}$ & $\begin{array}{l}\text { Adsorbed Amount } \\
\qquad \sigma\left(\mathbf{m g} / \mathbf{m}^{2}\right)\end{array}$ & $\begin{array}{c}\text { Surface Density } \\
\sigma\left(\mathrm{m}^{-2} \times 10^{-16}\right)\end{array}$ & $\begin{array}{c}1 /\left(\pi \mathbf{R}_{\mathrm{PS}}{ }^{2}\right) \\
\sigma_{\mathrm{ol}}\left(\mathbf{m}^{-2} \times 10^{-16}\right)\end{array}$ \\
\hline $3-36$ & 2.16 & 3.32 & 0.84 \\
\hline $9-36$ & 2.12 & 2.84 & 0.84 \\
\hline $16-31$ & 1.79 & 2.30 & 1.00 \\
\hline $18-36$ & 1.83 & 2.05 & 0.84 \\
\hline 15.92 & 2.02 & 1.13 & 0.27 \\
\hline $15-152$ & 1.97 & 0.71 & 0.15 \\
\hline $31-31$ & 1.66 & 1.61 & 1.00 \\
\hline $36-36$ & 1.49 & 1.25 & 0.84 \\
\hline $31-92$ & 1.82 & 0.89 & 0.27 \\
\hline $30-152$ & 1.89 & 0.63 & 0.15 \\
\hline $62-31$ & 1.36 & 0.88 & 1.00 \\
\hline $61-92$ & 1.65 & 0.65 & 0.28 \\
\hline $61-152$ & 2.05 & 0.58 & 0.15 \\
\hline $72-36$ & 1.67 & 0.93 & 0.84 \\
\hline $124-31$ & 1.57 & 0.61 & 1.00 \\
\hline t52-63 & 1.61 & 0.84 & 0.43 \\
\hline t9-32 & 1.95 & 2.90 & 0.98 \\
\hline $\mathrm{t} 124-60$ & 1.13 & 0.37 & 0.45 \\
\hline
\end{tabular}

\section{Sample}

PVP-PI

26-50

$1.25 \quad 0.39$

30-217

0.34

0.067

38-69

$1.00 \quad 0.26$

69-39

0.87

0.52 
which is geometry-independent and proportional to the energy per unit area of interaction between the polymer-bearing surfaces.

\section{RESULTS AND DISCUSSION}

\section{Adsorption measurement.}

Table I gives the measured adsorbed amounts for the range of block copolymers that we have studied. The data are given both as weight per unit area, $s$, and number of chains per unit area, $\sigma^{-2}$. The fourth column of Table I gives the values of $\sigma_{\mathrm{ol}}$ for each of the molecular weights of buoy block, calculated using Equation 1. All but the largest PVP molecular weights give $\sigma>\sigma_{\mathrm{ol}}$, so the vast majority of these layers have self-assembled into polymer brushes. An interesting observation is that the weight per unit area does not vary greatly over the range of copolymers we have studied.

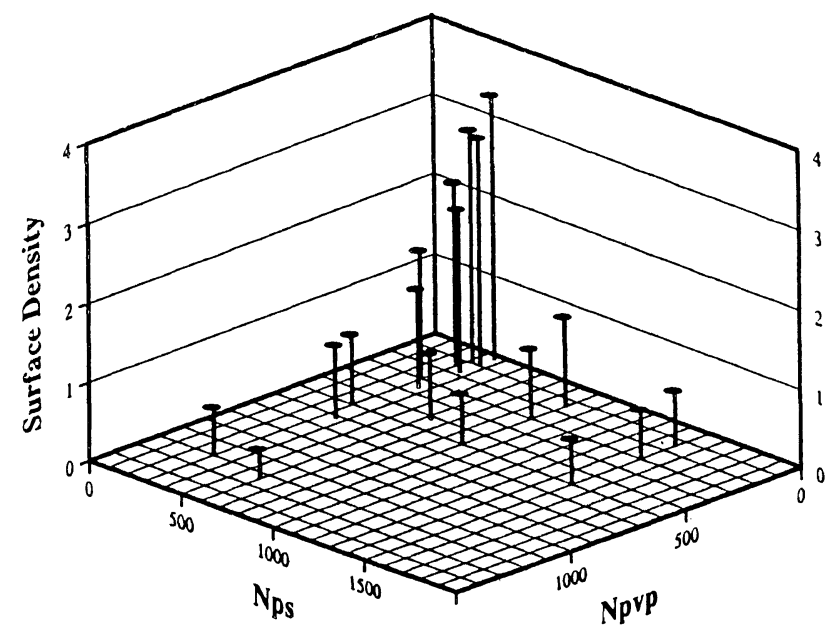

Figure 1 Measured surface densities $\sigma\left(\mathrm{m}^{-2} \times 10^{-16}\right)$ of Table 1 as a function of both the PS (NPS) and PVP (NPVP) degrees of polymerization. Vertical lines are used to indicate heights of data ponts above and their coordinates in NPS - NPVP plane.

Figure 1 displays these data as a 3-D perspective plot where $\sigma \mathrm{a}^{-2}$ is plotted versus both molecular weights of the two blocks. It is difficult to see clear trends in these data except that a fair generalization appears to be that the number of chains adsorbed per unit area decreases with increasing molecular weight of either of the two blocks. In order to derive more information from these data, we have attempted to find a combination of the two block molecular weights that would permit the collapse of the data as a function of both molecular weights to be represented as a single function of a combined molecular weight parameter. Using the simple ratio of the two block molecular weights does not work in a particularly impressive manner. What does work very well, as shown in Figure 2 , is to normalize $\sigma$ by $\sigma_{\mathrm{ol}}, \equiv \sigma^{*}$, and plot it as a function of what we shall term the "solvent-induced asymmetry" of the copolymer, $\beta \equiv \mathrm{N}_{\mathrm{B}}{ }^{6 / 5} / \mathrm{N}_{\mathrm{A}}{ }^{2 / 3}$, which is the square of the ratio of the swollen radius of the buoy block to the collapsed radius of the anchor block. This parameter reflects both the molecular weight and solvent quality asymmetry of the block copolymer. $\sigma^{*}$ is a measure of how crowded the buoys are.

Clearly the normalized number density of adsorbed chains is a unique function of this asymmetry parameter to a good degree of approximation. Furthermore, for all but the copolymers of highest asymmetry, $\sigma^{*}$ appears to be a power law function of $\beta$. That this 


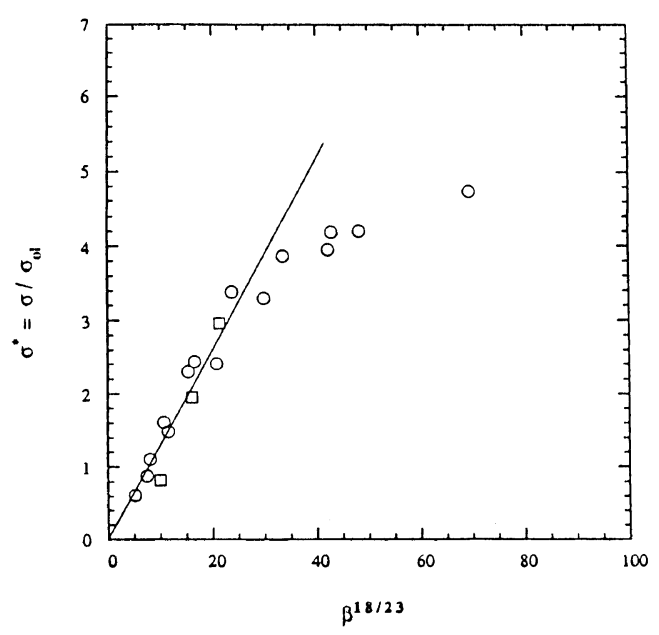

Figure 2 Normalized surface density $\sigma^{*}$ versus $\beta^{18 / 23}$ as measured by scintillation counting from tritium labeled adsorbed layers (squares) and XPS (circles). The solid line represents a least squares fit to the linear portion of the data (excluding five data points at highest $\beta$ values) as predicted from Equation 6

is reasonable can be argued from the theory of Marques, et $\mathrm{al}^{17}$, on the selective adsorption of block copolymers from selective solvents. They have analyzed the physics of this adsorption thoroughly and we present here a drastically simplified extract and minor modification of their theory.

We imagine the assembly of the layer from solution to be governed by a balance of only two energies. In this situation, the relevant energies are the adsorption energy of the anchor (favorable for adsorption) and the stretching energy of the brush (unfavorable for adsorption). Marques, et al, propose that the adsorption of the anchoring block takes place as though droplets of the material composing the anchor were wetting the surface and carrying along the buoy chains. This leads to an expression for the energy of the anchor layer that takes the form of a van der Waals energy, varying like the inverse square of the thickness of the layer, $\delta$, and containing an effective Hamaker constant that is a composite parameter of the relevant material Hamaker constants. Balancing this against the stretching energy of a layer containing $\sigma$ chains (Equation 4, assuming an athermal solvent for the buoy chains, so that $\chi=0$ or $v=1$ ) gives, for the assembly of the layer:

$$
\mathrm{F} / \mathrm{kT}=\mathrm{A} / 12 \mathrm{kT} \pi \delta^{2}+\mathrm{N}_{\mathrm{B}} \sigma^{11 / 6}
$$

The wetting anchor layer is a dense layer with a polymer volume fraction, $\phi=N_{A} a \sigma / \delta$, near unity, so $\delta \approx \mathrm{N}_{\mathrm{A}}$ a $\sigma$. Inserting this into Equation 5, and minimizing with respect to $\sigma$, then recasting the result as $\sigma^{*}$ as a function of $\beta$, gives:

$$
\sigma^{*} \approx(\mathrm{A} / \mathrm{kT})^{6 / 23} \beta^{18 / 23}
$$

For all but the copolymers of highest asymmetry, this equation is in good agreement with the data of Figure 2.

Clearly this curve must actually have a maximum at high asymmetry since this corresponds to negligible anchor block size and therefore to no adsorption $\left(\sigma^{*}=0\right)$. The lattice model theory of Evers, et al predicts this quantitatively ${ }^{18}$ and is, on preliminary examination, also able to explain some aspects of our data. This warrants a more 
thorough examination. We know empirically from our data that the deviation from power law behavior occurs at a number density of chains where the number of segments in the PVP blocks of those chains are in insufficient number to cover the surface completely, making the idea of a continuous wetting anchor layer untenable for these molecules. Bimodal brushes.

With the understanding we have developed for the unimolecular adsorption process, we can already suggest some expectations for equilibrium aspects of the assembly of bimodal brushes. For pairs of polymers such as the 61-92 and 61-152 samples of Table I, where the anchoring block molecular weight is the same and the adsorbed number densities in the pure layers are nearly the same, the major difference is in the stretching energy of the brush. Adsorption from a 50\% mole fraction solution should produce a layer where the stretching energy of the shorter component is largely unaffected where that of the longer component is favorably affected, relative to their respective pure component layers. Therefore, the total number density of chains should go up in the mixed layer.

In practice, the only bimodal brush layer on which we have data thus far is that composed of the samples 9-36 and 15-152 from Table I, where the measured surface densities of the pure component layers are reported. This is not an example of the idealized case described above. Experimentally, we find that adsorption from a $50 \mathrm{wt} \%$ solution of these two species at a total polymer concentration of $100 \mathrm{ppm}$ produces a layer that is $42 \mathrm{wt} \%$ of the 15-152 polymer, with a total number density, $\sigma_{\mathrm{T}} \mathrm{a}^{-2} \approx 1.5 \mathrm{x}$ $10^{16}$ chains $/ \mathrm{m}^{2}$. This is less than the arithmetic mean of the number densities of the pure component layers. Table II gives some other of the important characteristics of the mixed adsorbed layer. $\sigma_{\mathrm{m}} \mathrm{a}^{-2}$ is the number per unit area of that species in the mixed adsorbed layer.

Table II

Characteristics of Mixed Bimodal Adsorbed Layers

\section{Polymer component in the layer}

$15-152$

9-36

$$
\underline{\sigma}_{\mathrm{m}} \underline{\mathrm{a}}^{-2}\left(10^{16} \text { chains } / \mathrm{m}^{2}\right)
$$

1.24 $\underline{\sigma}_{\mathrm{ol}} \underline{\mathrm{a}}^{-2}\left(10^{16}\right.$ chains $\left./ \mathrm{m}^{2}\right)$

0.15

0.84

Both species in the mixed layer exceed their overlap densities, which is a sufficient condition for the remaining chain lengths of the long species to continue to overlap among themselves after emanating from the short brush. It is possible to adapt the arguments of Alexander ${ }^{6}$ and de Gennes ${ }^{7}$ to this type of bimodal brush. Before describing that analysis it is worth noting that all bimodal brushes are not necessarily of this category. It is also possible to make bimodal brushes with nonoverlapping in either the short or the long component, producing layers that are characterized by "underbrush" or "mop" structures, respectively. We shall deal only with the case where the long chains remain overlapped in the mixed layer, a structure we will call a "double brush", for simplicity.

The analysis of the double brush in the uniform stretching approximation of Alexander 6 and de Gennes ${ }^{7}$ is straightforward. The equation for the average thickness of the highest part of the mixed brush, analogous to Equation 3, is:

$$
\mathrm{L}_{\mathrm{m}}=\mathrm{N}_{\mathrm{S}} \mathrm{a}\left(\mathrm{v} \sigma_{\mathrm{T}}\right)^{1 / 3}\left[1+(\alpha-1) \gamma^{1 / 3}\right]
$$

where $\alpha=N_{L} / N_{S}$ and $\gamma=\sigma_{\mathrm{L}} / \sigma_{\mathrm{T}}$, and the subscripts $\mathrm{L}, \mathrm{S}$ and $\mathrm{T}$ refer to the long, short 
and total buoy chains, respectively.

Figure 3 presents data on measurements of force versus distance profiles between brushes assembled from the components of Table II. Data are shown for the two pure layers and for the mixed layer for which the relevant characteristics are given in Table II.

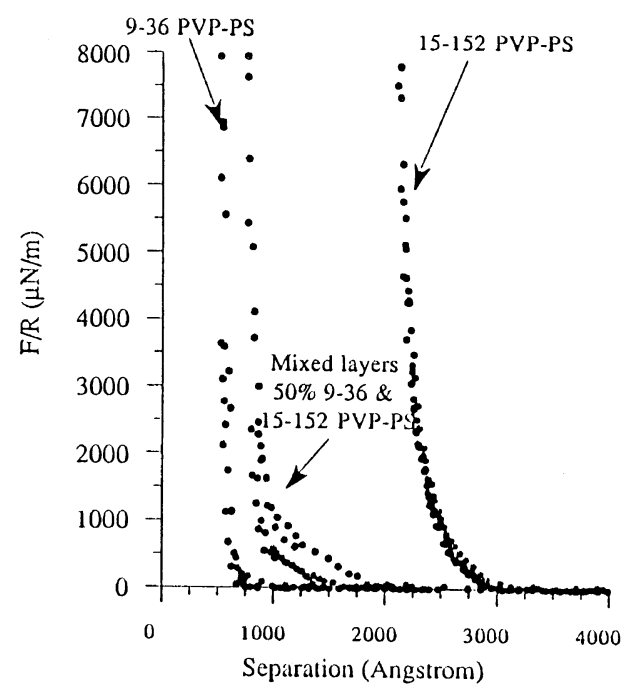

Figure 3 Force vs. distance profiles for pure and mixed layers of PVP-PS block copolymers $9-36$ and 15-152.

A complete quantitative analysis of these data is not yet complete but one can extract characteristic length scales from Figure 3 and compare them with expectations based on Equations 3 and 7. For the surface densities reported in Table I, Equation 3 predicts that the ratio of layer thicknesses, which we will take (somewhat arbitrarily) as half the distance at which the force rises to a value experimentally distinguishable from zero $(\approx 50 \mu \mathrm{N} / \mathrm{m}), \mathrm{L}_{\mathrm{L}} / \mathrm{L}_{\mathrm{S}}=\left(\mathrm{N}_{\mathrm{L}} / \mathrm{N}_{\mathrm{S}}\right)\left(\sigma_{\mathrm{L}} / \sigma_{\mathrm{S}}\right)^{1 / 3}$, which for the values given in Table I on these materials gives: $\mathrm{L}_{\mathrm{I}} / \mathrm{L}_{\mathrm{S}}=2.83$. Experimentally, the measured ranges of the forces have the ratio $\mathrm{L}_{\mathrm{U}} / \mathrm{L}_{\mathrm{S}} \approx 2950 / 900=3.27$, which is about $16 \%$ above the prediction. With this information on the pure layers as background it is of more interest to examine the range of the forces in the self-assembled mixed layers. From Equations 3 and 7, inserting the data from Tables I and II, the prediction for the ration of the mixed layer thickness to the pure short layer thickness is $\mathrm{L}_{\mathrm{m}} / \mathrm{L}_{\mathrm{S}}=2.26$. Experimentally, this ratio is $\mathrm{L}_{\mathrm{m}} / \mathrm{L}_{\mathrm{S}} \approx$ $1600 / 900=1.77$, which is about $21 \%$ below the prediction. Bear in mind that these predictions used experimental data on surface densities and so are not liable to errors in assumptions about the assembly of the layer (though of course they are liable to errors in the measurements of surfaces densities).

It appears reasonable to say at this point that the Alexander-de Gennes type of model for the bimodal brush gives a reasonable account of their characteristic length scales at a level of about $20 \%$ accuracy. Further work is required to strengthen this conclusion and to explore other interesting and important potential modifications of the profiles of polymer brushes.

In summary, it has been shown that self-assembly by selective adsorption of diblock copolymers from a selective solvent is an expedient means to produce a polymer brush. We have shown how the stretching engendered by the construction of the brush is an important determinant of the assembly process.Construction of bimodal double brushes and other mixed configurations will prove useful in tailoring profiles of segments. 


\section{REFERENCES}

1. G. Hadziioannou, S. Patel, S. Granick and M. Tirrell, J. Am. Chem. Soc., 108, 2869 (1986); M. Tirrell, S. Patel and G. Hadziioannou, Proc. Natl. Acad. Sci. USA, 84, 4725 (1987).

2. H.J. Taunton, C. Toprakcioglu and J. Klein, Macromolecules, 21, 3333 (1988).

3. E. E. Parsonage, M. Tirrell, H. Watanabe and R. G. Nuzzo, Macromolecules, 23, in press (1990).

4. W. B. Russel, D. A. Saville and W. R. Schowalter, "Colloidal Dispersions", Cambridge University Press, Cambridge, 1989.

5. L. L. Hench and D. R. Ulrich, eds., "The Science of Ceramic Chemical Processing", Wiley, N.Y., 1986.

6. S. Alexander, J. Phys. (Paris), 38, 983 (1977).

7. P.-G. de Gennes, Macromolecules, 13, 1069 (1980).

8. S. Patel, M. Tirrell and G. Hadziioannou, Colloids and Surfaces, 31, 215 (1988).

9. H.J. Taunton, C. Toprakcioglu, L.J. Fetters and J. Klein, Macromolecules, 23, 571 (1990).

10. S. Patel and M. Tirrell, Ann. Rev. Phys. Chem., 40, 597 (1989).

11. Philippe Auroy, "Polymères Greffés à l'Interface Solide-Liquide", Thèse, Université de Paris-Sud, 1990.

12. a) S. J. Hirz, "Modeling of Interactions between Adsorbed Block Copolymers", M.S. Thesis, University of Minnesota, 1986; S. T. Milner, T. A. Witten and M. E. Cates, Macromolecules, 21, 2610 (1988). b) M. Murat and G. S. Grest Macromolecules, 22, 4054 (1989).

13. P.-G. de Gennes, "Scaling Concepts in Polymer Physics", Cornell University Press, Ithaca, 1979, Chapter 2.

14. K. F. Freed, "Renormalization Group Theory of Macromolecules", Wiley, N.Y., 1987, pgs 66-70.

15. I. Noda, N. Kato, T. Kitano and M. Nagasawa, Macromolecules, 14, 668 (1981).

16. S. T. Milner, T. A. Witten and M. E. Cates, Macromolecules, 22, 853 (1989).

17. C. M. Marques, L. Leibler and J.F. Joanny, Macromolecules, 21, 1051 (1988).

18. O. A. Evers, J. M. H. M. Scheutjens and G. J. Fleer, J. Chem. Soc. Faraday Trans., 86, 1333 (1990). 